\title{
Decreased expression of the mitochondrial solute carrier SLC25A43 in basal cell carcinoma compared with healthy skin
}

\author{
SARA PROSÉN ${ }^{1}$, ANNA GÖTHLIN EREMO ${ }^{2}$, ALEXANDER DUARTE TSEGAI $^{3}$, \\ MAGNUS LINDBERG $^{1,3}$ and ELISABET TINA ${ }^{2}$ \\ ${ }^{1}$ Department of Dermatology, Örebro University Hospital, SE-701 85 Örebro; ${ }^{2}$ Department of Clinical Research Laboratory; \\ ${ }^{3}$ School of Medical Sciences, Faculty of Medicine and Health, Örebro University, SE-701 82 Örebro, Sweden
}

Received November 30, 2016; Accepted March 3, 2017

DOI: $10.3892 / \mathrm{ol} .2017 .6452$

\begin{abstract}
Basal cell carcinoma is the most common type of cancer in fair-skinned individuals, and its incidence is rapidly increasing. The aim of the present study was to investigate the gene and protein expression of the mitochondrial solute carrier family 25 member 43 (SLC25A43) in basal cell carcinoma. SLC25A43 has previously been identified to be genetically altered and associated with cell proliferation in human epidermal growth factor receptor 2-positive breast cancer. However, the knowledge about SLC25A43 is limited, and its role in other cancers is unknown. The SLC25A43 gene and protein expression was analysed in 14 basal cell carcinomas and healthy skin samples from the same individuals by quantitative polymerase chain reaction and immunohistochemistry, respectively. The results demonstrated a significantly lower $(\geq 50 \%)$ SLC25A43 gene expression in all carcinomas compared with that in healthy skin. In addition, SLC25A43 protein expression was absent in $>90 \%$ of all visual fields in the basal cell carcinomas, and the $\mathrm{H}$-score was significantly lower in tumours compared with the adjacent epidermis. These results demonstrate that SLC25A43 expression is altered at the gene and protein levels in basal cell carcinoma. The underlying mechanisms and the clinical relevance of these data must be elucidated in additional experimental and clinical studies.
\end{abstract}

\section{Introduction}

The incidence of skin cancer is rapidly increasing, and basal cell carcinoma is the most common type of cancer identified worldwide in fair-skinned individuals (1). There may also be a high number of unreported cases, since a number of tumours

Correspondence to: Dr Elisabet Tina, Department of Clinical Research Laboratory, Faculty of Medicine and Health, Örebro University, Campus USÖ, SE-701 82 Örebro, Sweden

E-mail: elisabet.tina@regionorebrolan.se

Key words: basal cell carcinoma, non-melanoma skin cancer, solute carrier family 25 member 43, quantitative polymerase chain reaction, immunohistochemistry are removed with local destructive methods and therefore are not histopathologically evaluated (2). Basal cell carcinoma incidence in Sweden is associated with an increasing age and is similar in men and woman (3). Metastasis is rare, but the tumours may occasionally grow in a local invasive manner with destruction of bone and cartilage (4). Due to its rising incidence, basal cell carcinoma is becoming a global public health problem and an economical burden for medical health care systems $(5,6)$. The requirement for simplified and cost-effective treatment options is therefore substantial (2). The development of vismodegib, a drug targeting the smoothened receptor in the hedgehog signalling pathway, has improved options for therapy in metastasising basal cell carcinoma (7). With the exception of the hedgehog signalling pathway, which is mutated in $\sim 85 \%$ of the basal cell carcinomas, mutations have been recognised in other genes, including tumour protein 53, v-myc avian myelocytomatosis viral oncogene neuroblastoma derived homolog, protein phosphatase 6 catalytic subunit and protein tyrosine phosphatase, non-receptor type 14 (8). Nevertheless, the underlying mechanisms for growth, progression and invasive capabilities of basal cell carcinoma are only partially recognised. The main risk factor for basal cell carcinoma is ultraviolet (UV) light exposure (9). The mitochondria are vulnerable to UVA light that induces oxidative damage $(10,11)$. Altered mitochondrial function is known to occur in malignant diseases, affecting cancer cell metabolism (12). However, the knowledge of the function of mitochondria in basal cell carcinoma is limited. Previous studies have identified deletion and altered methylation pattern of the mitochondrial solute carrier (SLC) gene SLC25A43, which is located at Xq24, in human epidermal growth factor receptor 2 (HER2)-positive breast tumours $(13,14)$. The deletion was also revealed to be present in cervix carcinoma and lung cancer in women (13). The biological function of SLC25A43 remains unknown, although the closest family members are transporters of coenzyme A and/or adenosine diphosphate $(15,16)$. An association between SLC25A43 protein expression and cell proliferation has been demonstrated in breast tumours, and gene silencing in vitro studies have observed that SLC25A43 protein expression affects the cell proliferation rate of breast cancer cells (17). However, there are no previous studies on SLC25A43 in basal cell carcinoma, and considering that men have only one copy of chromosome $\mathrm{X}$, the expression may differ between sexes. 
The aim of the present study was to determine the gene and protein expression of SLC25A43 in basal cell carcinomas compared with that in healthy skin from the gluteal area in the same individuals, both in men and women.

\section{Patients and methods}

Patients. A total of 14 patients, ( 7 men and 7 women) with a suspected basal cell carcinoma were included. Patients with recurrent tumours or tumours from where biopsies had already been obtained for diagnostic purpose were excluded from the study. The patient age ranged from 64 to 92 years, and the size of the tumours varied from 8 to $20 \mathrm{~mm}$ in diameter. The tumours were excised from the face $(n=7)$ or trunk $(n=7)$. The histopathological evaluation of the included tumours exhibited a nodular growth pattern $(n=11)$, mixed superficial/nodular $(n=1)$ and aggressive growth pattern $(n=2)$.

At the time of excision, a $4 \mathrm{~mm}$ in diameter-punch biopsy was obtained from the centre of the tumour and from healthy (normal) gluteal skin from the same patient. Tissue samples from the two first patients were snap frozen using dry ice with isopropanol, while samples from the rest of the patients were placed in Allprotect Tissue Reagent (Qiagen $\mathrm{GmbH}$, Hilden, Germany). All samples were stored at $-80^{\circ} \mathrm{C}$. The present study was approved by the local Ethics Committee in Uppsala, Sweden (Uppsala/Örebro approval no. 2011/242) with requirement of informed and written consent from all the participants. All samples, including patient information, were stored and handled in encoded format.

Quantitative polymerase chain reaction ( $q P C R)$. The frozen tissues were homogenised using TissueLyserII (Qiagen $\mathrm{GmbH}$ ) with $5 \mathrm{~mm}$ steel beads (Qiagen $\mathrm{GmbH}$ ) twice for $2 \mathrm{~min}$ at $20 \mathrm{~Hz}$. RNA extraction was performed using AllPrep DNA/RNA/Protein Mini kit (Qiagen $\mathrm{GmbH}$ ) according to the manufacturer's protocol. Concentrations of total RNA were measured with NanoDrop ND-1000 UV-Vis Spectrophotometer (NanoDrop Technologies; Thermo Fisher Scientific, Inc., Wilmington, DE, USA). A total of 75 ng RNA was converted to complementary (c)DNA using High-Capacity cDNA Reverse Transcription kit (Applied Biosystems; Thermo Fisher Scientific, Inc., Waltham, MA, USA) in a $20 \mu \mathrm{l}$ reaction. The expression of the target gene SLC25A43 (Hs00933775_m1) and the two reference genes ABL1 (Hs01104728_m1) and $\beta$-actin (NM_001101.2; all from Applied Biosystems; Thermo Fisher Scientific, Inc.) was measured with TaqMan Gene Expression assays in duplicates. To each well, $1.5 \mu \mathrm{l}$ cDNA was added to a final volume of $15 \mu \mathrm{l}$, and qPCR was performed first at $50^{\circ} \mathrm{C}$ for $2 \mathrm{~min}$ and $95^{\circ} \mathrm{C}$ for $20 \mathrm{sec}$, followed by 40 cycles of $95^{\circ} \mathrm{C}$ for $1 \mathrm{sec}$ and $60^{\circ} \mathrm{C}$ for $20 \mathrm{sec}$ using the 7900HT Fast Real-Time PCR System (Applied Biosystems; Thermo Fisher Scientific, Inc.). The cycle thresholds values were automatically set in the SDS 2.4 software (Applied Biosystems; Thermo Fisher Scientific, Inc.). SLC25A43 gene expression in the samples was normalised against the mean quantification cycle $(\mathrm{Cq})$ value of the two reference genes $\left(2^{-\Delta \mathrm{Cq}}\right)$, and fold-change values were obtained using the $2^{-\Delta \Delta \mathrm{Cq}}$ method (18).

Immunohistochemistry. Formalin-fixed and paraffin-embedded tumour biopsies were obtained and sliced into $4 \mu \mathrm{m}$-thick sections. In order to optimize the protocol, liver and gallbladder were used as positive-staining controls. Tissue samples from colon were used as a negative control, while samples from healthy skin were used to estimate the baseline protein expression. Tissues for routine quality control were provided by the Department of Pathology, Örebro University Hospital (Örebro, Sweden). They were selected based on information from the antibody manufacturer. Deparaffinisation and antigen retrieval were performed using Decloaking Chamber (Biocare Medical, Pacheco, CA, USA) and Borg Decloaker, RTU (Biocare Medical) for $10 \mathrm{~min}$ at $110^{\circ} \mathrm{C}$. Unspecific binding sites and endogenous peroxidase activity were blocked for $5 \mathrm{~min}$ in room temperature (RT) followed by $30 \mathrm{~min}$ incubation at RT with anti-SLC25A43 antibody (cat. no. HPA035188; dilution, 1:175; Sigma-Aldrich; Merck KGaA, Darmstadt, Germany) in Da Vinci Green Diluent (Biocare Medical). The slides were incubated with MACH 1 Universal HRP-Polymer (Biocare Medical) for $30 \mathrm{~min}$ at RT and visualised with 3,3'-diaminobenzidine for $5 \mathrm{~min}$ at RT. The staining was performed using an intelliPATH FLX ${ }^{\mathrm{TM}}$ Automated Slide Staining System and reagents from the MACH 1 Universal HRP-Polymer Detection system (both from Biocare Medical). Subsequent to counterstaining with Mayer's haematoxylin for $5 \mathrm{~min}$ at RT, the slides were dehydrated in increasing concentrations of ethanol followed by xylene, prior to mounting with PERTEX ${ }^{\circledR}$ mounting medium (Histolab, Stockholm, Sweden). Samples from liver and healthy skin were used as positive and negative staining controls, respectively.

Ten vision fields at $\times 40$ magnification were observed in epidermis and tumour areas, and scored from 0 to $3+(0$, negative expression; $1+$, low expression; $2+$, intermediate expression; and $3+$, high expression) using an Olympus BX41 microscope. Each individual slide was scored twice by one observer (A.D.T.), and a second observer (S.P.) evaluated the results. A final score (0-30) was obtained using an alteration of the H-score (19), by multiplying the number of vision fields within each score $(0,1,2$ and 3$)$ and then the values were added together [ $(1 \mathrm{x}$ (number of vision fields $1+)+2 x$ (number of vision fields $2+$ ) + $3 \mathrm{x}$ (number of vision fields $3+$ )]. The slides were scanned with the digital slide scanner Pannoramic 250 Flash III (3DHistech, Ltd., Budapest, Hungary), and micrographs were captured using the CaseViewer version 2.0 software (3DHistech, Ltd.).

Statistical analysis. The differences in gene expression between tumour and healthy tissues were calculated by paired sample t-test using normalised $\mathrm{Cq}$ values $\left(2^{-\Delta \mathrm{Cq}}\right)$. To evaluate the difference in fold-change $\left(2^{-\Delta \Delta \mathrm{Cq}}\right)$ and the difference in $\mathrm{H}$-score between the sexes, the Mann-Whitney U-test was used. In order to calculate the difference in SLC25A43 protein expression between adjacent epidermis and basal cell carcinoma, a Wilcoxon signed rank test was used for associated samples. The statistical analyses were performed using SPSS 23.0 statistical software for Windows (IBM SPSS, Armonk, NY, USA) and GraphPad Prism version 6.0 (GraphPad Software, Inc., La Jolla, CA, USA). P $\leq 0.05$ was considered to indicate a statistically significant difference.

\section{Results}

Gene expression of SLC25A43. The gene expression of SLC25A43 was analysed in basal cell carcinomas and healthy 
gluteal skin in 14 individuals. All tumours exhibited lower gene expression compared with healthy skin $(\mathrm{P}<0.05$; Fig. $1 \mathrm{~A})$, with a fold-change ranging 0.50 to 0.17 , equivalent to a $50-83 \%$ decrease. There was no difference in gene expression between sexes ( $\mathrm{P}=0.81$; Fig. 1B).

Protein expression of SLC25A43. SLC25A43 protein expression was assessed in 13 out of 14 tumours and in the tissue controls. The positive controls (liver and gallbladder) exhibited the expected staining according to the antibody supplier (Sigma-Aldrich; Merck KGaA) and The Human Protein Atlas (20). The staining intensity and the fraction of stained cells in healthy skin samples were generally low, although cytosolic immunoreactivity was observed in the basal cell layer of epidermis. The immunoreactivity displayed an irregular pattern, with a heterogeneous intensity that varied from 0 to $3+$. The dermis, including structures in the hair follicle, exhibited minimal staining. Staining was absent in the negative control (colon).

The evaluation of SLC25A43 protein expression in the tumour tissues demonstrated that the basal cells of epidermis in the edges of the tumours exhibited more intense staining than the atrophic epidermis located directly above the basal cell carcinoma, similar to the staining of samples from healthy skin. In total, $>50 \%$ of the visual fields in the adjacent epidermis revealed different degrees of positivity, while $>90 \%$ of all visual fields in tumour tissues were negative (Fig. 2). The $\mathrm{H}$-score was significantly lower in tumours (median $\mathrm{H}$-score, 0; range, 0-4) than in adjacent epidermis (median $\mathrm{H}$-score, 9; range, 0-16; $\mathrm{P}<0.05)$. There was no difference in protein expression between sexes $(\mathrm{P}=0.54)$.

\section{Discussion}

In the present study, the expression of the inner membrane mitochondrial SLC25A43 in basal cell carcinoma was investigated. All the basal cell carcinomas in the present study exhibited lower SLC25A43 gene expression in comparison with that of the gluteal skin of the study subjects. The protein level of SLC25A43 in tumour tissues was lower compared with that in adjacent epidermis, as assessed by immunohistochemistry. The mitochondria are mainly involved in cell energy turnover (21). Various tumour cells exhibit an altered metabolism in comparison with that of healthy cells, using glycolysis over oxidative phosphorylation (i.e., the Warburg effect) $(12,22)$. As the tumour cells utilise more glucose than healthy cells in the surrounding tissue, they have an advantage in cell division and growth. It may also facilitate the tumour cells to escape the mitochondrial-dependent steps of reactive oxygen species-mediated apoptosis and the mitochondrial-dependent regulation of the cell cycle (23). The knowledge about mitochondrial regulation of the cell cycle has increased during the last decade $(24,25)$. A previous study revealed increased proliferation of HER2-positive breast cancer cells when SLC25A43 gene expression was silenced (17). This result indicates a role for SLC25A43 as a regulator of cell cycle progression. In a previous study on basal cell carcinoma compared with healthy skin, several mitochondrial genes involved in metabolism were identified to be downregulated (26). In that study, the analysis was limited to the expression of 1,700 genes, and the authors did not report SLC25A43 as one of their results (26).

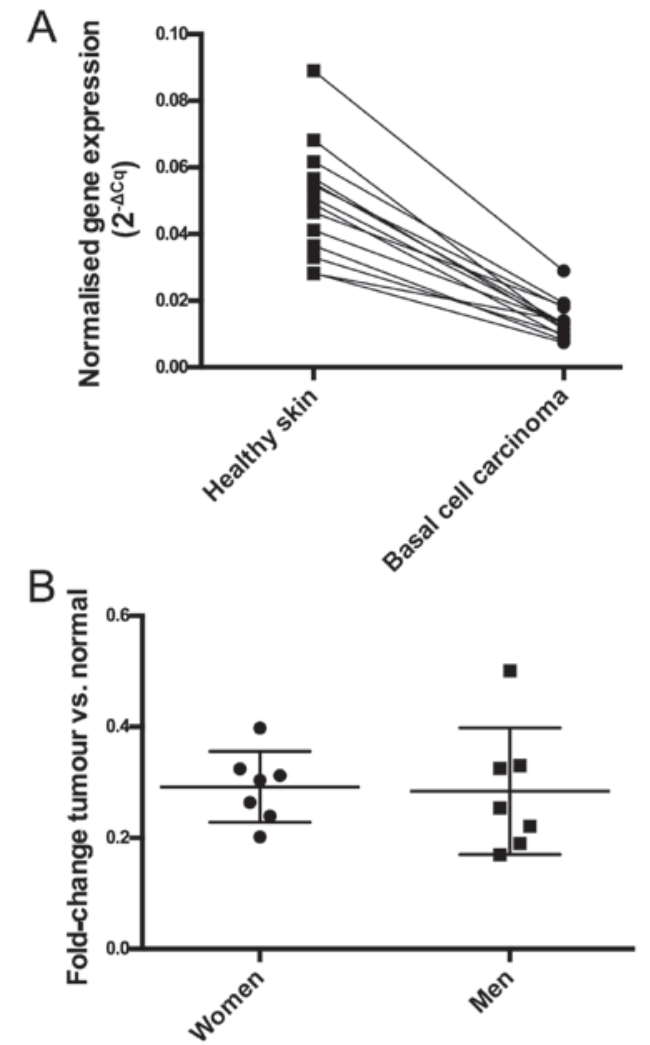

Figure 1. SLC25A43 gene expression. (A) Normalized gene expression of SLC25A43 in healthy skin and basal cell carcinoma $(\mathrm{P}<0.05)$. (B) Fold-change $\left(2^{-\Delta \Delta C q}\right)$ of SLC25A43 in women and men $(\mathrm{P}=0.81)$. Cq, quantification cycle; SLC25A43, solute carrier family 25 member 43 .

In the present study, protein expression was assessed using, to the best of our knowledge, the only commercially available antibody against SLC25A43. The antibody was previously evaluated by The Human Protein Atlas (20), and this description of staining was used as a reference in the present study. According to The Human Protein Atlas, which considered the intensity of staining and the fraction of stained cells, SLC25A43 does not appear to be detected in skin. However, their staining results appear to be similar to those of the present study, with scattered positive cells in the basal layer of epidermis. Thus, the protein expression of SLC25A43 in healthy skin tissue is low, and it appears that basal cell carcinomas express even lower levels. The Human Protein Atlas' results suggest a high gene expression of SLC25A43 in the skin, but the antibody used in that study failed to demonstrate high protein expression (27). In the present study, a difference in protein expression between normal epidermis and basal cell carcinoma was observed, even though the staining intensity was low.

In the present study, SLC25A43 staining was localised to the cytoplasm in the basal cell layer in the epidermis, where the energy consumption is expected to be high due to cell division. The cells in the basal cell layer with the highest staining were scattered in concordance with the epidermis proliferation pattern. In the hair follicles, there was almost no visible staining. The origin of basal cell carcinoma is important to discuss. The tumour cells exhibit histological similarities to the basal layer in the epidermis, but immunochemistry and genetic analyses reveal similarities to the follicular epithelia $(26,28)$. In the present 
A

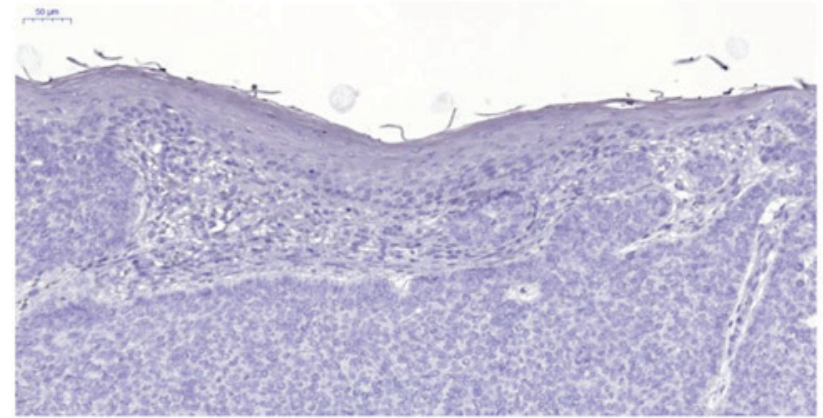

C

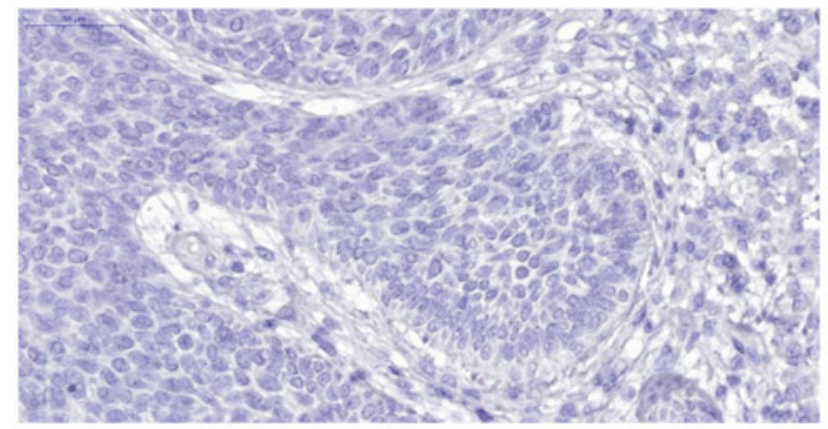

E

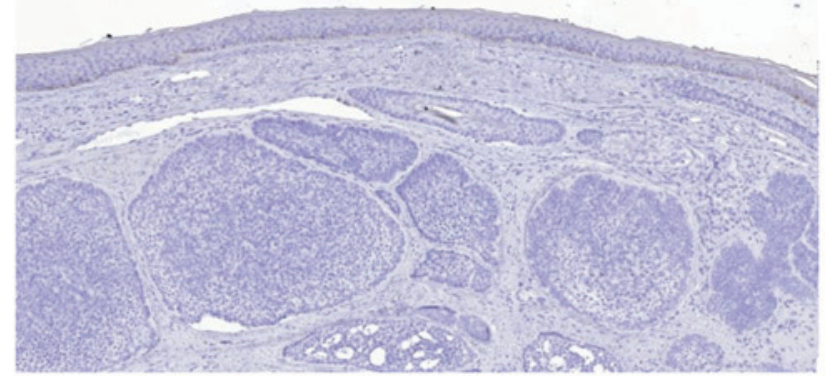

B

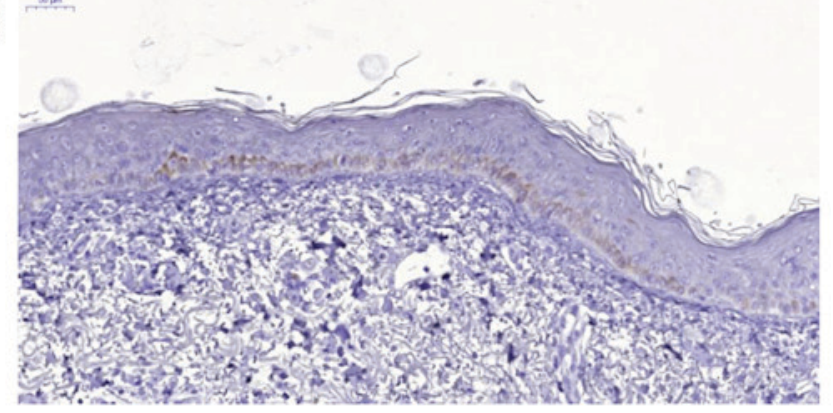

D

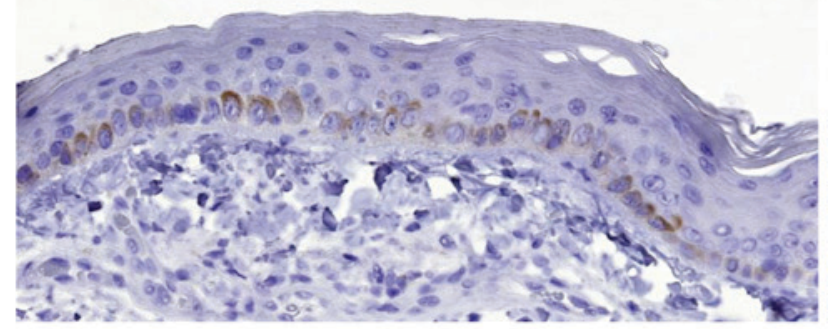

$\mathrm{F}$

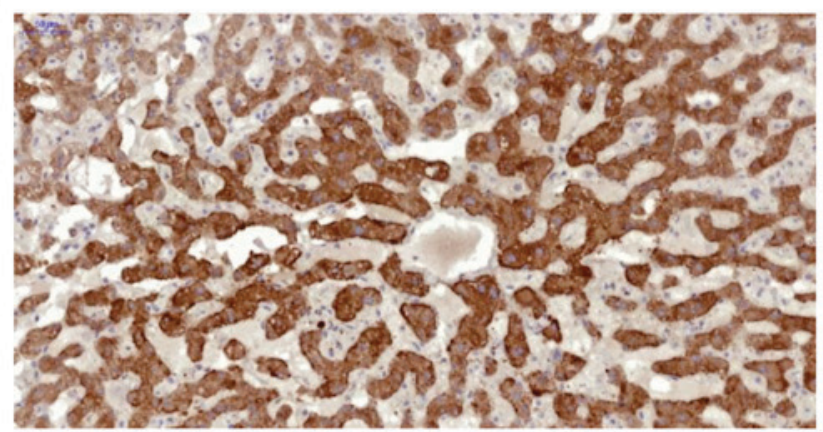

Figure 2. Immunohistochemical staining of solute carrier family 25 member 43. Micrographs of the epidermis located (A) directly above a basal cell carcinoma and (B) at the edge of the tumour exhibit different staining intensity (magnification, x20). (C) Cells in a basal cell carcinoma and (D) cells in the adjacent epidermis (magnification, $\mathrm{x} 40$ ). (E) Basal cell carcinoma (magnification, $\mathrm{x} 10$ ) and (F) liver tissue, which served as a positive-staining control (magnification, $\mathrm{x} 20)$.

study, basal cell carcinoma and follicular epithelia exhibited almost no staining. Additional studies on SLC25A43 expression in the skin are required to evaluate whether decreased expression of SLC25A43 in basal cell carcinoma compared with in healthy skin tissues indicates a functional pattern that reflects the metabolic activity of the cells or the cells' origin. There is also a need to further elucidate if SLC25A43 participates in the process of carcinogenesis and if other factors serve important roles.

The exact mechanism or consequence of the altered expression of SLC25A43 in basal cell carcinoma is unknown. Nevertheless, the present study revealed a distinct difference between tumours and healthy skin. Previous studies have reported a deletion in the SLC25A43 gene in HER2-positive breast cancers, in addition to cervix and lung cancer in women (13). These results suggest that the loss of SLC25A43 promotes tumour cell growth. Epigenetic X chromosome inactivation in women implies that an alteration in only one allele will be sufficient for a loss in gene function. SLC25A43, located on the X chromosome, has not been studied previously in male cancer; however, the analyses of the present study did not conclude any differences in gene or protein expression between men and women. Although the present study includes a low number of participants, the incidence of basal cell carcinoma is almost equal for men and women, indicating that there are no sex-specific gene alterations.

In conclusion, the present study has demonstrated a significantly lower gene and protein expression of SLC25A43 in basal cell carcinomas compared with that in healthy skin. In order to identify improved and novel treatment strategies, the understanding of the mechanisms behind the progression of basal cell carcinoma is essential. The data of the present study indicate that SLC25A43 may be altered in basal cell carcinoma; however, additional studies are required to elucidate its actual role and importance.

\section{Acknowledgements}

The authors thank Ms. Hanna Arnesson (Clinical Research Laboratory, Faculty of Medicine and Health, Örebro University, Sweden) for her methodological assistance. The present study was supported by a grant from Nyckelfonden (OLL-255231), Örebro University Hospital Cancer Foundation, Sweden. 


\section{References}

1. Lomas A, Leonardi-Bee J and Bath-Hextall F: A systematic review of worldwide incidence of nonmelanoma skin cancer. Br J Dermatol 166: 1069-1080, 2012.

2. Holm AS, Nissen CV and Wulf HC: Basal cell carcinoma is as common as the sum of all other cancers: Implications for treatment capacity. Acta Derm Venereol 96: 505-509, 2016.

3. Socialstyrelsen: Cancer i siffror 2013. http://www.socialstyrelsen. se/publikationer2013/2013-6-5. Accessed April 27, 2015.

4. Wong CS, Strange RC and Lear JT: Basal cell carcinoma. BMJ 327: 794-798, 2003.

5. Housman TS, Feldman SR, Williford PM, Fleischer AB Jr, Goldman ND, Acostamadiedo JM and Chen GJ: Skin cancer is among the most costly of all cancers to treat for the Medicare population. J Am Acad Dermatol 48: 425-429, 2003.

6. Gordon LG and Rowell D: Health system costs of skin cancer and cost-effectiveness of skin cancer prevention and screening: A systematic review. Eur J Cancer Prev 24: 141-149, 2015.

7. Sekulic A, Migden MR, Oro AE, Dirix L, Lewis KD, Hainsworth JD, Solomon JA, Yoo S, Arron ST, Friedlander PA, et al: Efficacy and safety of vismodegib in advanced basal-cell carcinoma. N Engl J Med 366: 2171-2179, 2012.

8. Bonilla X, Parmentier L, King B, Bezrukov F, Kaya G, Zoete V, Seplyarskiy VB, Sharpe HJ, McKee T, Letourneau A, et al: Genomic analysis identifies new drivers and progression pathways in skin basal cell carcinoma. Nat Genet 48: 398-406, 2016.

9. Moan J, Grigalavicius M, Baturaite Z, Dahlback A and Juzeniene A: The relationship between UV exposure and incidence of skin cancer. Photodermatol Photoimmunol Photomed 31: 26-35, 2015.

10. Birch-Machin MA and Swalwell H: How mitochondria record the effects of UV exposure and oxidative stress using human skin as a model tissue. Mutagenesis 25: 101-107, 2010.

11. Lu J, Sharma LK and Bai Y: Implications of mitochondrial DNA mutations and mitochondrial dysfunction in tumorigenesis. Cell Res 19: 802-815, 2009.

12. Hanahan D and Weinberg RA: Hallmarks of cancer: The next generation. Cell 144: 646-674, 2011

13. Tina E, Lindqvist BM, Gabrielson M, Lubovac Z, Wegman P and Wingren S: The mitochondrial transporter SLC25A43 is frequently deleted and may influence cell proliferation in HER2-positive breast tumors. BMC Cancer 12: 350, 2012.

14. Lindqvist BM, Farkas SA, Wingren S and Nilsson TK: DNA methylation pattern of the SLC25A43 gene in breast cancer. Epigenetics 7: 300-306, 2012.
15. Haitina T, Lindblom J, Renström T and Fredriksson R: Fourteen novel human members of mitochondrial solute carrier family 25 (SLC25) widely expressed in the central nervous system. Genomics 88: 779-790, 2006.

16. Fiermonte G, Paradies E, Todisco S, Marobbio CM and Palmieri F: A novel member of solute carrier family 25 (SLC25A42) is a transporter of coenzyme $\mathrm{A}$ and adenosine 3 ',5'-diphosphate in human mitochondria. J Biol Chem 284: 18152-18159, 2009.

17. Gabrielson M, Reizer E, Stål O and Tina E: Mitochondrial regulation of cell cycle progression through SLC25A43. Biochem Biophys Res Commun 469: 1090-1096, 2016.

18. Livak KJ and Schmittgen TD: Analysis of relative gene expression data using real-time quantitative PCR and the 2(-Delta Delta C(T)) Method. Methods 25: 402-408, 2001

19. Detre S, Saclani Jotti G and Dowsett M: A ‘quickscore' method for immunohistochemical semiquantitation: Validation for oestrogen receptor in breast carcinomas. J Clin Pathol 48: 876-878, 1995 .

20. The Human Protein Atlas: version 16.1. http://www.proteinatlas. org/ENSG00000077713-SLC25A43/antibody. Accessed March 9, 2017.

21. Osellame LD, Blacker TS and Duchen MR: Cellular and molecular mechanisms of mitochondrial function. Best Pract Res Clin Endocrinol Metab 26: 711-723, 2012.

22. Warburg O, Wind F and Negelein E: The metabolism of tumors in the body. J Gen Physiol 8: 519-530, 1927.

23. Martinez-Reyes I and Cuezva JM: The H(+)-ATP synthase: A gate to ROS-mediated cell death or cell survival. Biochim Biophys Acta 1837: 1099-1112, 2014.

24. Sakamaki T, Casimiro MC, Ju X, Quong AA, Katiyar S, Liu M, Jiao X, Li A, Zhang X, Lu Y, et al: Cyclin D1 determines mitochondrial function in vivo. Mol Cell Biol 26: 5449-5469, 2006.

25. Van den Bogert C, Muus P, Haanen C, Pennings A, Melis TE and Kroon AM: Mitochondrial biogenesis and mitochondrial activity during the progression of the cell cycle of human leukemic cells. Exp Cell Res 178: 143-153, 1988.

26. Howell BG, Solish N, Lu C, Watanabe H, Mamelak AJ, Freed I, Wang B and Sauder DN: Microarray profiles of human basal cell carcinoma: Insights into tumor growth and behavior. J Dermatol Sci 39: 39-51, 2005.

27. The Human Protein Atlas: version 16.1. http://www.proteinatlas. org/ENSG00000077713-SLC25A43/tissue. Accessed March 9, 2017.

28. Asada M, Schaart FM, de Almeida HL Jr, Korge B, Kurokawa I, Asada Y and Orfanos CE: Solid basal cell epithelioma (BCE) possibly originates from the outer root sheath of the hair follicle. Acta Derm Venereol 73: 286-292, 1993. 